\title{
A Possible Test for Ground-State Correlations in Superfluid Nuclei.
}

\author{
O. Civitarese (*), S. M. Peltier (**) and A. Plastino (***) \\ Facultad de Ciencias Exactas, Universidad Nacional de La Plata - La Plata \\ E. S. HERNANDEZ (***) \\ Facultad de Ciencias Exactas y Naturales, Universidad de Buenos Aires - Buenos Aires
}

(ricevuto il 24 Settembre 1974)

During the last years the zero-point vibrations associated with the ground-state correlations (g.s.c.) have received considerable attention (1). Most of this work, however, has been devoted to normal systems, i.e. nonsuperconducting ones. Only recently g.s.c. in superfluid nuclei have been studied in a rather detailed way $\left(^{2-4}\right)$. These investigations suggest that effects unambiguously attributable to g.s.c. should be very hard to observe. Stripping and pick-up processes might yield direct experimental evidence of g.s.c. However, opposite to what happens in the case of normal systems, the theoretical figures involved strongly indicate that neither in deformed heavy nuclei $\left(^{3}\right)$ nor in single-closed-shell ones $\left({ }^{4}\right)$ is the situation amenable to successful experimental study.

It could be argued that the different results obtained for energies and transition rates either with the quasi-particle random phase approximation (QRPA) or with the quasi-particle Tamm-Dancoff one (QTDA), constitute an evidence of the effects of g.s.c. However it could be also argued that, in nuclear-structure calculations, both the force constant and the effective charge include effects of the configuration space and, in this sense, a suitable choice of this space and the observation of the Pauli principle in each stage of the corresponding evaluation would eventually make different methods to agree as to the final results. It should then be very desirable to find some observable direct and appreciably affected by g.s.c. It is the purpose of this note to suggest that electromagnetic-transition rates between excited states may strongly show the influence of g.s.c. In a pioneering paper ARviEu and SALUSTI $\left(^{5}\right)$ pointed out the difficulty inherent

\footnotetext{
(*) Fellow of the CONICET, Argentina.

(**) Fellow of the CICPBA, Argentina.

(***) Member of the Carrera del Investigador, CONICET, Argentina.

(1) A. Goswami and M. K. Pal: Nucl. Phys., 44, 294 (1963); E. A. Sanderson: Phys. Lett., 19,

141 (1965); D. Agassi, V. Gilitet and A. Lombroso: Nucl. Phys,, 130 A, 129 (1969); E. Gmitrova,

M. Gmiro and J. K. Gambhir: IC/70/146 (preprint).

(2) F. S. Hernandez and A. Plistino: Phys. Lett., 39 B, 163( 1972).

$\left(^{3}\right.$ E. S. Hernandez and A. Plastino: Zeits. Phys., 268, 337 (1974).

$\left({ }^{4}\right)$ S. M. Pemtier, A. Plastino and E. S. Hernandez: Zeits. Phys. (to be published).

$\left({ }^{5}\right)$ R. ARvieu and F. Salusti: Nucl. Phys., 66, 305 (1963).
} 
to the QRPA method in what concerns the evaluation of this kind of transitions, which heretofore will be referred to as interband ones. The basic difference between the QTDA and the QRPA lies in the explicit consideration, in the latter, of the so-called backward going graphs $\left({ }^{6.7}\right)$. In computing matrix elements corresponding to transitions to the ground-state (g.s.) it is not necessary to know the detailed structure of the correlated g.s. But, as remarked by ARVIEU and SALUSTI ( ${ }^{5}$ ), this information is essential in order to evaluate interband transitions. This difficulty can be overcome by explicit reference to the structure of the QRPA g.s., which can be constructed by the action upon the BCS wave function of an operator $\exp [S]$ defined as $\left({ }^{6}\right)$

$$
\exp [S]=\exp \left[\sum_{\substack{i k k m \\ J M}} C_{i k l m}^{J}\left[b_{i}^{+} b_{l k}^{+}\right]^{J M}\left[b_{l}^{+} b_{m}^{+}\right]^{\overline{J M}}\right] .
$$

The sum in (1) runs over pairs $i, k ; l, m$ of quasi-particle operators $b$ which couple to angular momentum $J$, projection $M$ and parity.

All states characterized by these last quantum numbers are to be considered. The index $n$ is used to distinguish among them. The $C$ 's are the so-called correlation coefficients. They can be obtained from the solutions of the QRPA eigenvalue problem for excited states of energy $w\left({ }^{7}\right)$

$$
\left[\begin{array}{rr}
P & Q \\
-Q & -P
\end{array}\right]\left[\begin{array}{l}
X \\
Y
\end{array}\right]=w\left[\begin{array}{l}
X \\
Y
\end{array}\right]
$$

by means of a relationship which in matrix notation can be stated as $\left({ }^{6}\right)$

$$
Y=X C
$$

The $X$ 's are the so-called forward going amplitudes, while the $Y$ 's give the corresponding backward going ones, which are a distinctive feature of the QRPA formalism.

Let $T(k q)$ be the one-particle operator attached to the emission of multipolar electric radiation of angular momentum $k$ and component $q$. One can evaluate its matrix element for a transition between two states of the form $\left(^{5}\right)$

$$
\left.|n J M\rangle=\sum_{i l}\left[X_{i l}^{J}\left(b_{i}^{+} b_{l}^{+}\right)^{J M}+(-)^{J+M} Y_{i l}^{J}\left(b_{\imath} b_{l}\right)^{J-M}\right] \mid \mathrm{QRPA}(\text { g.s. })\right\rangle
$$

by explicitly expressing the ground-state according to (1)

$$
|\mathrm{QRPA}(\mathrm{g} . \mathrm{s} .)\rangle=\exp [S]|\mathrm{BCS}\rangle
$$

A very involved expression results, after some tedious Wick's algebra, which one may present with some computing difficulties. A detailed account will in this respect be given in a fortheoming paper. However, results can easily be obtained in the case of a simple model $\left(^{8}\right)$ which has become very fashionable for testing now approaches in nuclear physics $\left({ }^{9}\right)$. We consider the case of 2 particles interacting via the surface

( ${ }^{\circ}$ D. J. Rowe: Nuclear Collective Motion (London, 1970).

(?) M. Baranger: Phys. Rev., 120, 957 (1960).

$\left({ }^{8}\right)$ J. Hogasen-Feldian: Nucl. Phys., 28, 258 (1961).

(") See, for example, H. LifKIN, N. Mezkov and A. GLICK: Nucl. Phys., 62, 188 (1965). 
delta-interaction (SDI) $\left({ }^{10}\right)$ filling two shells of opposite parity, each of a degeneracy $2 \Omega$ and separated by an energy gap $2 \varepsilon$. Let us choose $\varepsilon=1 \mathrm{MeV}$ and $\Omega=6$. We adopt here the results of ref. $\left.{ }^{11}\right)$ and solve the corresponding equations according to the schematic approach of BRown and BosterLy $\left({ }^{12}\right)$. In the two-shell model, for states of positive parity, the $O$-matrix of eq. (2) is a $2 \times 2$ one. Moreover, it can be easily seen that the symmetries involved here result in $O$-matrices in which the diagonal elements vanish identically. These facts enormously simplify the problem. Notice also that only those $C$ 's for the $J$ 's involved in the transition will enter the calculations. One can, in addition, verify that in this simple model the normalization condition makes the structure of the corresponding wave functions independent of the value chosen for the SDI coupling constant $k$. As a consequence, matrix elements for the interband transitions do not depend on the value of the coupling constant, opposite to what happens, of course, with the excitation energies arising as roots of the well-known secular equation $\left({ }^{12}\right)$.

We have thus evaluated the $B(E 2)$ value for the transition between a $4^{+}$- and a $2^{+}$-state in this model. This has been done in two different ways. In the first one we perform a QTDA calculation, in which no g.s.c. are considered. We call the $B(E 2)$ value thus computed the uncorrelated one. On the other hand we perform the $Q R P A$ evaluation, taking into account the full expression for the QRPA g.s. We call this the correlated value. We obtain the result

$$
\frac{B(E 2)_{\text {correlated }}-B(E 2)_{\text {unoorrolated }}}{B(E 2)_{\text {uncorrelated }}}=0.57
$$

A percentage of $57 \%$ should be appreciable enough to be experimentally observed. If the results of this simple model constitute an indication of what happens in more involved situations, interband transitions may yield direct evidence on g.s.c. in superconducting nuclei, and should be subjected, therefore, to further study in more realistic cases.

$\left({ }^{10}\right)$ A. Plastino, R. Anvieu and S. M. Moskowski: Phys. Rev., 145, 837 (1966).

(ii) H. Vucetich, A. Plastino and F. Kripotic: Zeits. Phys., 220, 218 (1966).

(12) G. E. Brown and M. Bosteri. : Phys. Rev. Lett., 3, 472 (1959). 